\title{
Agrobacterium tumefaciens ExoR represses succinoglycan biosynthesis and is required for biofilm formation and motility
}

\author{
Amelia D. Tomlinson, Bronwyn Ramey-Hartung, $†$ Travis W. Day, \\ Peter M. Merritt and Clay Fuqua
}

Correspondence

Clay Fuqua

cfuqua@indiana.edu

Received 12 February 2010

Revised 15 June 2010

Accepted 21 June 2010

\author{
Department of Biology, Indiana University, Bloomington, IN 47405, USA
}

The ubiquitous plant pathogen Agrobacterium tumefaciens attaches efficiently to plant tissues and abiotic surfaces and can form complex biofilms. A genetic screen for mutants unable to form biofilms on PVC identified disruptions in a homologue of the exoR gene. ExoR is a predicted periplasmic protein, originally identified in Sinorhizobium meliloti, but widely conserved among alphaproteobacteria. Disruptions in the $A$. tumefaciens exoR gene result in severely compromised attachment to abiotic surfaces under static and flow conditions, and to plant tissues. These mutants are hypermucoid due to elevated production of the exopolysaccharide succinoglycan, via derepression of the exo genes that direct succinoglycan synthesis. In addition, exoR mutants have lost flagellar motility, do not synthesize detectable flagellin and are diminished in flagellar gene expression. The attachment deficiency is, however, complex and not solely attributable to succinoglycan overproduction or motility disruption. A. tumefaciens ExoR can function independently of the ChvG-Chvl two component system, implicated in ExoR-dependent regulation in $S$. meliloti. Mutations that suppress the exoR motility defect suggest a branched regulatory pathway controlling succinoglycan synthesis, motility and biofilm formation.

\section{INTRODUCTION}

Agrobacterium tumefaciens is a well-studied plant pathogen best known for its ability to genetically transform host plants (Escobar \& Dandekar, 2003). A. tumefaciens is a member of the family Rhizobiaceae and, although a pathogen, is closely related to nitrogen-fixing plant symbionts. The process of interkingdom gene transfer in A. tumefaciens is controlled in response to plant exudates, including certain phenolics and sugars, and requires a type IV secretion system (for reviews, see Christie et al., 2005; Gelvin, 2003). Infection and gene transfer are dependent on attachment of the bacteria to the plant surface. Attachment and biofilm formation by A. tumefaciens C58 have been well established. Indeed, after initial attachment A. tumefaciens can assemble into complex biofilms on plant

tPresent address: PGXL Laboratories, 201 E. Jefferson Street, Suite 309, Louisville, KY 40202, USA.

‡Present address: Department of Pharmacy, Wishard Health Services, 1001 West 10th Street, Indianapolis, IN 46202, USA.

Abbreviations: CV, crystal violet; GPCR, quantitative PCR; SDCM, spinning disk confocal microscopy.

A supplementary figure, showing alignment of ExoR sequences, and a supplementary table, listing oligonucleotide sequences used for molecular cloning and directed mutagenesis, are available with the online version of this paper. tissues and abiotic surfaces (Danhorn et al., 2004; Merritt et al., 2007; Ramey et al., 2004).

Many bacteria efficiently adhere to surfaces, including a wide range of animal and plant pathogens. In these systems attachment is often mediated by cell-surface adhesins such as pili, flagella, curli and polysaccharides. Rhizobial attachment to plants is a critical early step in symbiosis, and probably involves several proteinacious adhesins, including the so-called Rap adhesins and rhicadhesin (Ausmees et al., 2001; Russo et al., 2006; Smit et al., 1989), as well as cell-surface polysaccharides (Laus et al., 2006). No analogous cell-surface adhesins have been definitively reported for A.tumefaciens, although it appears to produce a rhicadhesin-type molecule (Dardanelli et al., 2003). Flagellar motility is required for efficient attachment of $A$. tumefaciens to a variety of surfaces, and non-motile mutants show deficiencies in pathogenesis (Chesnokova et al., 1997; Merritt et al., 2007). Several genes reported to be required for attachment and virulence, encoded within the Att cluster (Matthysse et al., 2000), have been subsequently shown to be dispensable for these processes (Nair et al., 2003). In general, little is known about the factors required for attachment or their coordination during the transition of A. tumefaciens to the sessile phase.

Biofilms can range from flat featureless layers to topographically complex assemblages, with cells adhering to 
surfaces and cohering to other cells (Hall-Stoodley et al., 2004). The cells within biofilms are often enmeshed within a matrix largely composed of exopolysaccharide, but which may also contain proteins and nucleic acids (Branda et al., 2005). Clonal growth of adherent cells, surface-based motility and matrix production drive the formation of mature biofilms. This maturation can be controlled at multiple points through a variety of regulatory pathways that respond to changing environmental conditions. In several different bacteria, regulators of exopolysaccharide biosynthesis have profound effects on the eventual structure and composition of the mature biofilm. For example, VpsR of Vibrio cholerae represses the Vibrio polysaccharide (vps) genes; mutants for this regulator overproduce Vps and form dense (rugose) biofilms, in comparison to the more diffuse wild-type biofilms (Lim et al., 2007). In the plant vascular pathogen Pantoea stewartii, synthesis of the exopolysaccharide stewartan is controlled by the EsaR-EsaI quorum sensing system, which also regulates biofilm formation (Koutsoudis et al., 2006). In Sinorhizobium meliloti, regulation of the exopolysaccharide succinoglycan influences the microbe's ability to form symbiotic nodules with host legumes. Several studies have described a potential role for succinoglycan in biofilm formation by $S$. meliloti, though a recent report has called this conclusion into question (Fujishige et al., 2006; Rinaudi \& Gonzalez, 2009; Wells et al., 2007; Yao et al., 2004). A. tumefaciens, like S. meliloti, also produces succinoglycan (Cangelosi et al., 1987).

In A. tumefaciens, several regulators have been shown to influence attachment and biofilm formation (Fuqua, 2008). The FNR-type transcription factor SinR affects late stages of biofilm maturation, possibly in response to oxygen limitation (Ramey et al., 2004). BigR, a repressor originally identified in Xylella fastidiosa, is conserved in $A$. tumefaciens; bigR mutants form abnormally dense biofilms on abiotic surfaces (Barbosa \& Benedetti, 2007). Phosphorus limitation also results in enhanced biofilm formation through the PhoR-PhoB two-component regulatory system (Danhorn et al., 2004).

In this study, we isolated an A. tumefaciens transposon mutant on the basis of its profound attachment deficiency. The transposon insertion disrupts the regulator exoR, previously shown to control succinoglycan production in S. meliloti (Reed et al., 1991; Yao et al., 2004). Null mutations in the A. tumefaciens exoR gene result in elevated succinoglycan levels, reduced flagellar motility, prevention of surface attachment and complete loss of biofilm formation.

\section{METHODS}

Strains, plasmids, reagents and growth conditions. All strains and plasmids used in this study are described in Table 1. Buffers, antibiotics and media were obtained from Fisher Scientific and Sigma. DNA manipulations were performed in accordance with standard protocols (Sambrook et al., 1989). DNA sequencing was performed with ABI Big Dye Terminator version 3.1 on an ABI 3730 sequencer operated by the Indiana Molecular Biology Institute. Oligonucleotides (see Supplementary Table S1, available with the online version of this paper) were obtained from Integrated DNA Technologies. Plasmids were electroporated into A. tumefaciens by a standard method (Mersereau et al., 1990). A. tumefaciens derivatives were grown in AT minimal salts medium and $15 \mathrm{mM}\left(\mathrm{NH}_{4}\right) \mathrm{SO}_{4}$ and either $1 \%(\mathrm{w} / \mathrm{v})$ glucose (ATGN) or $0.4 \%(\mathrm{w} / \mathrm{v})$ succinic acid (ATSucN) as the carbon source (Tempé et al., 1977). Antibiotics were used at the following concentrations $\left(\mu \mathrm{g} \mathrm{ml}^{-1}\right)$ : for A. tumefaciens, ampicillin (Ap) 50, gentamicin $(\mathrm{Gm})$ 500; kanamycin (Km) 150; spectinomycin (Sp) 50; streptomycin (Sm) 2000; and for Escherichia coli, Ap 100, Gm 25, Km 25, Sp 50. Antibiotic selection was maintained throughout all experiments when using mutants generated by plasmid insertion. Calcofluor was added to media at $200 \mu \mathrm{g} \mathrm{ml}^{-1}$. Swim agar contained $0.2,0.25$ or $0.3 \%$ BactoAgar.

Identification of the exoR gene. The A. tumefaciens $\sin 2$ and $\sin 3$ mutants were isolated using a transposon mutagenesis screen described previously (Ramey et al., 2004). A transposon mutant library was generated using the pTnMod-OKm' plasposon system (Dennis \& Zylstra, 1998). Two thousand mutants were evaluated using a microtitre plate adhesion assay (O'Toole et al., 1999). Genomic DNA from these mutant isolates was purified and digested with BamHI or SacII, and fragments were ligated under dilute conditions to favour self-ligation. The resulting plasmids were transformed en masse into E. coli $\mathrm{DH} 10 \mathrm{~B} / \lambda$ pir. Plasmids isolated from $\mathrm{Km}^{\mathrm{R}}$ colonies carried the plasposon insertion and flanking genomic DNA. The resulting plasmids were designated pSin 2 and pSin3, and were sequenced using primers complementary to the transposon ends.

Molecular cloning and directed mutagenesis. In-frame deletions of the exoR and exoA genes were introduced via allelic replacement as described previously (Merritt et al., 2007). Sequences flanking each side of the target gene were amplified by PCR, fused by standard ligation and cloned into pGEM-T (Promega) at which time the inserts were confirmed by sequencing. The resulting deletion fragments were excised by restriction digestion and ligated into suicide vector pKNG101 (Kaniga et al., 1991). For exoR, the oligonucleotides exoRupfwd and exoRuprev were used to amplify the upstream fragment, and exoRdownfwd and exoRdownrev were used for the downstream fragment; for exoA oligonucleotides ExAUSS and ExAUSAS were used to amplify the upstream fragment, and ExADSS plus ExADSAS were used for the downstream fragment. The resulting plasmids were conjugated into A. tumefaciens C58 from the SM10/2pir donor, and prototrophic $\mathrm{Sm}^{\mathrm{R}}$ transconjugants were isolated on minimal media. Deletion mutants of the resident gene generated by excision of the plasmid were allowed to accumulate by culturing those derivatives that carried the integrated plasmid for several generations without antibiotic selection. Cultures were then plated on ATGN solid media, and colonies were selected for sucrose resistance. Candidates were purified and confirmed as deletion mutants by PCR amplification across the deletion and sequencing of the PCR product.

A plasmid-borne copy of the exoR gene was constructed by amplifying the exoR coding sequence by PCR and cloning into pBBR1-MCS5, generating pTWD100. The exoR coding sequence on pTWD100 was sequenced to ensure that it was free of mutations and properly fused to $\mathrm{P}_{l a c}$

The $\operatorname{ch} v$ gene was mutagenized by plasmid integration. An internal 520 bp fragment of the $\operatorname{chvG}$ coding region (codons 154-329) was amplified using oligonucleotides $\operatorname{ch} v G$ trunc fwd and $\operatorname{ch} v G$ trunc rev, and cloned into pCR2.1-TOPO. The insert was excised as an EcoRI/ $X b a \mathrm{I}$ fragment and ligated into pVIK112 (Kalogeraki \& Winans, 1997) to generate pADT112. The resulting plasmid was conjugated from the E. coli Sm10/Apir donor into C58, $\Delta$ exoR, $\triangle$ exoA and $\triangle$ exoRexoA strains. Integrants were selected for $\mathrm{Km}^{\mathrm{R}}$ on minimal 
Table 1. Bacterial strains and plasmids

\begin{tabular}{|c|c|c|}
\hline Strain or plasmid & Relevant characteristics & Reference \\
\hline \multicolumn{3}{|l|}{ A. tumefaciens } \\
\hline C58 & Nopaline-type strain, pTiC58, pATC58 & Sciaky et al. (1978) \\
\hline $\sin 2$ & exoR::TnMod-OKm', $\mathrm{Km}^{\mathrm{R}}$ & This work \\
\hline $\operatorname{Sin} 3$ & exoR::TnMod-OKm', $\mathrm{Km}^{\mathrm{R}}$ & This work \\
\hline BER9 & exoR::pRR100 & This work \\
\hline PMM1 & $\Delta \operatorname{exoR}$ C58 derivative & This work \\
\hline PMM2 & $\Delta$ exoRexoA C58 derivative & This work \\
\hline PMM4 & $\Delta f l g E$ C58 derivative & Merritt et al. (2007) \\
\hline ADT1 & $\operatorname{chvG::pADT112}$ & This work \\
\hline ADT2 & 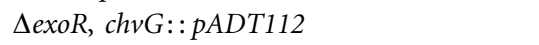 & This work \\
\hline ADT3 & 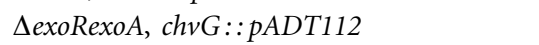 & This work \\
\hline ADT10 & Class I suppressor of PMM1 & This work \\
\hline ADT11 & Class II suppressor of PMM1 & This work \\
\hline ADT12 & Class I suppressor of PMM2 & This work \\
\hline \multicolumn{3}{|l|}{ E. coli } \\
\hline S17-1/2pir & $\lambda$ pir, $\mathrm{Tra}^{+}$, cloning host & Gift of V. de Lorenzo \\
\hline SM10/ipir & $\lambda$ pir, cloning host & Miller et al. (1989) \\
\hline $\mathrm{DH} 10 \mathrm{~B} / \lambda$ pir & $\lambda$ pir, cloning host & Gift of David Bauer \\
\hline $\mathrm{DH} 5 \alpha \mathrm{F}^{\prime}$ & Cloning host & Woodcock et al. (1989) \\
\hline TOP10 & Cloning host & Invitrogen \\
\hline \multicolumn{3}{|l|}{ Plasmids } \\
\hline pTNMod-OKm' & Plasposon & Dennis \& Zylstra (1998) \\
\hline pGEM-T & Cloning vector & Promega \\
\hline pCR2.1-TOPO & Cloning vector & Invitrogen \\
\hline pKNG101 & Cloning vector, $\mathrm{Suc}^{\mathrm{S}} \mathrm{Sp}^{\mathrm{R}}$ & Kaniga et al. (1991) \\
\hline pJZ383 & $\mathrm{P}_{t a c}:: g f p, \mathrm{Sp}^{\mathrm{R}}, \mathrm{pVS}$ replicon & J. Zhu; Cormack et al. (1996) \\
\hline pRR100 & exoR mutation vector, $\mathrm{pVIK} 112$ derivative & This work \\
\hline pTD100 & $\mathrm{P}_{l a c}::$ exoR, pBBR1MCS-5 derivative & This work \\
\hline pTWD114 & exoA deletion vector, pKNG101 derivative & This work \\
\hline pADT112 & $\operatorname{chv} G$ mutation vector, $\mathrm{pVIK} 112$ derivative & This work \\
\hline
\end{tabular}

media, and disruption of the $\operatorname{ch} v G$ coding region was confirmed by PCR amplification and sequencing. The site of integration results in a ChvG protein truncated at amino acid 329, prior to the histidine kinase and HATPase domains.

Sequences of all oligonucleotides used for molecular cloning and directed mutagenesis are available in Supplementary Table S1.

Static culture biofilm assays. Static culture biofilms were grown on sterile PVC coverslips suspended vertically in the wells of UV-sterilized 12 -well polystyrene dishes. Cultures were inoculated at $\mathrm{OD}_{600} 0.05$ in $3 \mathrm{ml}$ ATGN broth and incubated at room temperature for 24-48 h. Adherence to PVC coverslips was visualized by staining with crystal violet $(\mathrm{CV})$. Quantification of adherent biomass was achieved via solubilization of adsorbed CV in a standardized volume of $33 \%$ acetic acid. Solubilized CV was measured as $A_{600}$ to estimate relative amounts of adhered biomass. To visualize $\beta$-linked polysaccharides in adherent biomass, inoculated coverslips were washed in deionized water and stained in $1 \%$ calcofluor ( $\mathrm{w} / \mathrm{v}$ double distilled water).

Flow cell culture biofilms: growth and analysis. Flow cell biofilms were cultured as described previously (Ramey et al., 2004). Flow cells were purchased through the Technical University of Denmark and inoculated with diluted overnight cultures $(100 \mu \mathrm{l}$ per flow chamber, final $\mathrm{OD}_{600}$ 0.05). After inoculation, cells were permitted to attach for $60 \mathrm{~min}$ before the flow of ATGN media with appropriate antibiotics commenced at $\sim 3 \mathrm{ml} \mathrm{h}^{-1}$. Each strain was inoculated into triplicate chambers, and growing biofilms were examined by spinning disk confocal microscopy (SDCM) at $12 \mathrm{~h}$ intervals, beginning $24 \mathrm{~h}$ post-inoculation and continuing to 5 days post-inoculation. Microscopy was performed with a Nikon TE-200 spinning disk confocal microscope at $100 \times$ magnification fitted with a Uniblitz shutter driver and an Orca ER camera (Hamamatsu). SDCM images were acquired with the Perkin-Elmer ULTRAVIEW software package and analysed using an automated version of biofilm analysis software COMSTAT (Heydorn et al., 2000) called autoCOMSTAT, running in MATLAB 7.0 (Merritt et al., 2007).

Plant attachment assays. Attachment assays with Arabidopsis thaliana ecotype WS used 7-10-day-old seedlings grown from ethanol-surface-sterilized seeds planted on half-strength MurashigeSkoog agar media. Roots were cut into $1 \mathrm{~cm}$ segments and transferred to sterile dishes containing $2 \mathrm{ml} 1 \mathrm{mM} \mathrm{CaCl}_{2}$ and $0.4 \%$ sucrose, and inoculated to $\mathrm{OD}_{600} 0.01$ with the appropriate derivative strain of $A$. tumefaciens C58 carrying a green fluorescent protein (GFP) reporter construct under constitutive expression $\left(P_{\mathrm{tac}}:: g f p\right)$. Four root segments were inoculated per strain. After a given period of incubation in the dark at room temperature, the root segments were recovered, rinsed and resuspended in fresh calcium chloride/sucrose solution, and sealed under coverslips for hydration. Epifluorescence microscopy was performed on a Nikon E800 at $60 \times$ magnification with METAMORPH software.

Quantification of extracellular polysaccharide production. To quantitatively compare levels of secreted exopolysaccharide between strains, a standard anthrone assay was implemented (Morris, 1948). 
This assay measures the total reducing sugar content in a given sample. Cultures were grown in AT media containing $0.4 \%$ succinic acid as the carbon source to avoid addition of extraneous reducing sugars. At mid-exponential growth phase, samples were prepared by centrifugation to remove cells. This cell-free culture fluid was assayed for exopolysaccharide content via sulfuric acid hydrolysis in the presence of the colorimetric indicator anthrone. To assay, $250 \mu \mathrm{l}$ anthrone solution $(2 \% \mathrm{w} / \mathrm{v}$ in ethyl acetate) was added to $1 \mathrm{ml}$ cellfree culture fluid, then hydrolysed with $2.5 \mathrm{ml}$ concentrated sulfuric acid. $A_{620}$ was measured and normalized to the $\mathrm{OD}_{600}$ of each culture.

Quantitative PCR (qPCR). Primers were designed to amplify approximately $150 \mathrm{bp}$ sequences from the upstream regions of the exoY (Atu3327) and flgD (Atu0579) reading frames. Primer sequences are included in Supplementary Table S1. Cultures were grown in ATGN media with the appropriate antibiotics to an $\mathrm{OD}_{600}$ of approximately 0.8 , and RNA was isolated using Qiagen RNAprotect reagent and RNEasy Mini Prep kits. Samples were treated with DNase (Ambion) to remove contamination. cDNA was prepared using qScript cDNA Super Mix kit (Quanta Biosciences). qPCR was performed with PerfeCta SYBR Green FastMix Low Rox reagent (Quanta Biosciences), on a Stratagene MX3000P instrument. Samples values were normalized using a $\sigma^{70}$ primer set and calibrated against wild-type results. Data are representative of two biological replicates, each of which consisted of three technical replicates.

\section{RESULTS}

To identify genes required for surface colonization, we screened a set of 2000 A. tumefaciens C58 transposon mutants for decreased adherence to 96-well PVC microtitre plates. Among the mutants isolated were two designated $\sin 2$ and $\sin 3$ (surface interaction deficient). These two mutants were severely impaired in biofilm formation on abiotic surfaces, and were strikingly hypermucoid when grown on solid medium (Fig. 1a-d). They are prototrophic, with growth rates identical to those of the wildtype in AT minimal medium.

The $\sin 2$ and $\sin 3$ mutants carry unique transposon insertions in Atu1715 (Goodner et al., 2001; Wood et al., 2001), with the $\sin 2$ insertion at codon 253 and the $\sin 3$ insertion at codon 215. This gene encodes a homologue of the ExoR protein of Sinorhizobium meliloti. ExoR is a negative regulator of exopolysaccharide synthesis in several rhizobia and is conserved among a wide range of alphaproteobacteria, including both plant and animal symbionts as well as free-living micro-organisms (see Supplementary Fig. S1, available with the online version of this paper). The ExoR sequence contains a series of tetratricopeptide repeat and SEL1 domains, sequence motifs implicated in protein-protein interactions (Blatch \& Lassle, 1999; D'Andrea \& Regan, 2003). Additionally, the ExoR sequence suggests the presence of an N-terminal signal sequence, and periplasmic localization has been demonstrated for ExoR of S. meliloti (Wells et al., 2007). ExoR has no recognizable DNA-binding domains, but its expression is known to affect the transcript levels of target exo genes involved in synthesis of $S$. meliloti succinoglycan (Reed et al., 1991; Yao et al., 2004) as well as affecting transcription of flagellar genes (Yao et al., 2004), primarily
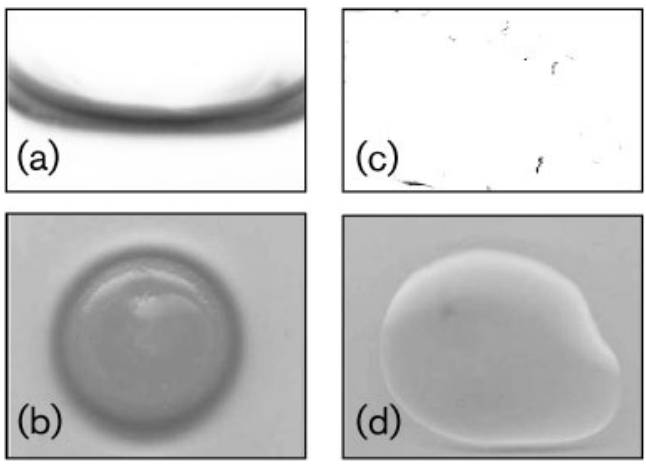

(e)

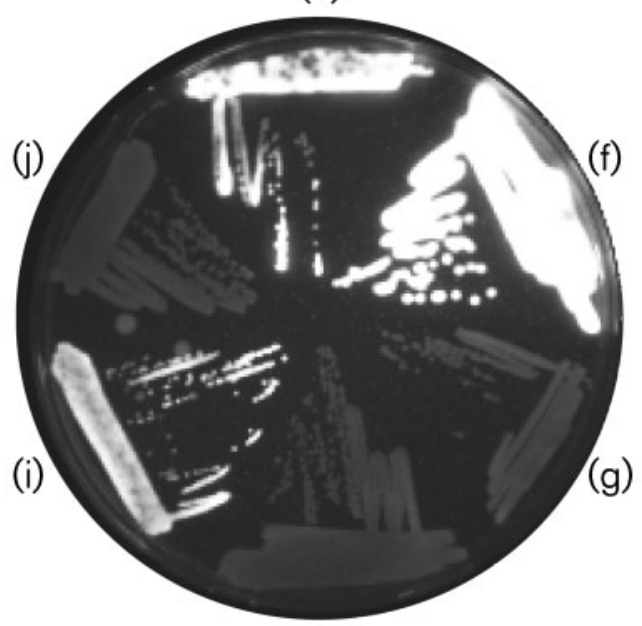

(h)

Fig. 1. Biofilm and mucoidy phenotypes of the A.tumefaciens $\sin 3$ mutant and derivatives. (a)-(d) Wild-type A. tumefaciens C58 forms a biofilm on PVC coverslips (a), and a normal colony on minimal media (b), while the $\sin 3$ mutant fails to form a biofilm on PVC coverslips (c) and is hypermucoid on solid media (d). (e)-(j) A. tumefaciens derivatives streak-isolated on ATGN medium with $1 \%$ Calcofluor demonstrate differential production of $\beta$-linked polysaccharides. Wild-type fluorescence is visible in (e), while the hyperfluorescent exoR mutant can be seen in (f). The exoA mutant (g) is dim, as is the exoRexoA mutant (h). Addition of $\mathrm{P}_{\text {lac }}::$ exoR complements the hyperfluorescence of the exoR mutant (i), but not the dimness of the exoRexoA mutant (j). exoR mutant strains used in this assay were generated via Campbell integration.

through interactions with the ExoS-ChvI two component system (Chen et al., 2008; Wells et al., 2007). The hypermucoid phenotype of the $S$. meliloti exoR mutant, caused by overproduction of succinoglycan, was the reason for its initial isolation (Reed et al., 1991).

Site-directed disruption of exoR using plasmid integration mutagenesis with an internal exoR fragment (C58-JR1), and independently by complete deletion of the gene (C58PMM1), yielded prototrophic mutants with biofilm and hypermucoid phenotypes identical to the original transposon mutants. Complementation with an intact, plasmid- 
borne copy of exoR expressed from the lac $Z$ promoter ( $\mathrm{pTWD100}, \mathrm{P}_{\text {lac }}:$ exoR) effectively reversed these phenotypes. Except where noted, all results shown for the exoR mutant are from the complete deletion mutant C58PMM1.

\section{Overproduction of succinoglycan in the $A$. tumefaciens exoR mutant}

Based on the findings from S. meliloti, it seemed likely that the hypermucoid phenotype observed in the exoR mutants was due to overproduction of succinoglycan. Accordingly, we examined the effect of abolishing succinoglycan synthesis in wild-type and exoR mutant backgrounds. ExoA has been characterized as a glycosyltransferase involved in addition of the first glucose in the octasaccharide repeat subunit during succinoglycan biosynthesis in $S$. meliloti (Reuber \& Walker, 1993). The exoA gene was disrupted by an unmarked, non-polar deletion of the entire coding sequence to eliminate succinoglycan production in A. tumefaciens. A. tumefaciens derivatives were plated on standard minimal medium containing $1 \%$ calcofluor, a fluorescent dye that binds to $\beta$-linked polysaccharides such as succinoglycan and fluoresces under UV light (Doherty et al., 1988). Compared with wild-type cells (Fig. 1e), exoR mutant cells are hyperfluorescent, indicating the elevated production of $\beta$-linked polysaccharides (Fig. 1f), while exoA mutant cells are significantly less fluorescent than wild-type cells (Fig. 1g). The exoRexoA derivative has fluorescence equivalent to the exo $A$ mutant and is nonmucoid, suggesting that the hypermucoidy and bright calcofluor fluorescence of the exoR mutant are dependent on the exo genes, and therefore primarily due to the overproduction of succinoglycan (Fig. 1h).

To determine relative levels of exopolysaccharide production, we evaluated each mutant using an anthrone-based assay. Soluble polysaccharides from cell-free supernatants were hydrolysed with sulfuric acid in the presence of the colorimetric indicator anthrone that detects reducing sugars (Morris, 1948). The exoR mutants produce approximately fourfold greater amounts of soluble exopolysaccharide than wild-type, whereas exoA and exoRexoA mutants produce roughly one-third of the wild-type amount during exponential-phase growth (Table 2).

\section{Disruption of exoR elevates expression of the succinoglycan biosynthetic gene exo $Y$}

To determine whether ExoR regulates succinoglycan production via expression of exo genes, we performed qPCR on the exoY transcript in A. tumefaciens C58, $\Delta$ exoR, C58 $\left(\mathrm{P}_{l a c}::\right.$ exoR $)$ and $\Delta$ exoR $\left(\mathrm{P}_{\text {lac }}::\right.$ exoR $)$ strains. This analysis revealed a striking 30 -fold derepression of exoY expression in the exoR mutant that was fully reversed by provision of the plasmid-borne exoR. Expression of ExoR from this same plasmid in a wild-type background led to a slight reduction in exoY expression compared with the wild-type strain (Table 3). Analysis of an exoT-lacZ fusion in these same strains showed a similar, but slightly reduced, pattern of expression (data not shown). These findings demonstrate that $A$. tumefaciens ExoR exerts a significant negative regulation on the expression of exo genes, which probably explains the observed overproduction of succinoglycan in an exoR mutant.

\section{The exoR adherence defect is not dependent on succinoglycan synthesis}

The most striking visible phenotype of the exoR mutant is its hypermucoidy (Fig. 1). We hypothesized that the elevated production of exopolysaccharide reduced the efficiency or duration of surface interactions, yielding the observed biofilm deficiency. To test this directly, the exoR and exoA mutants were assayed for biofilm formation on the model abiotic surface PVC in a standard static culture biofilm assay. The exoA mutant revealed no attachment

Table 2. Biofilm and exopolysaccharide phenotypes of mutant derivatives

\begin{tabular}{|c|c|c|}
\hline Strain & Biofilm $A_{600} / \mathrm{OD}_{600} *$ (SEM) & Exopolysaccharide $A_{620} / \mathrm{OD}_{600}(\mathrm{SEM}) \dagger$ \\
\hline C58 & $1.81(0.18)$ & $3.02(0.16)$ \\
\hline$\triangle e x o R$ & $0.31(0.04)$ & $12.21(0.40)$ \\
\hline$\triangle e x o A$ & $1.17(0.08)$ & $0.94(0.09)$ \\
\hline$\Delta$ exoRexoA & $0.23(0.04)$ & $1.03(0.34)$ \\
\hline $\operatorname{chvG}$ & $2.85(0.15)$ & $2.25(0.17)$ \\
\hline $\operatorname{chvG} \Delta \operatorname{exoR}$ & $0.21(0.03)$ & $5.90(0.24)$ \\
\hline $\operatorname{chvG} \triangle \operatorname{exoA}$ & $1.38(0.07)$ & $1.75(0.39)$ \\
\hline $\operatorname{chv} G \Delta \operatorname{exoRexoA}$ & $0.17(0.03)$ & $0.74(0.63)$ \\
\hline$\Delta e x o R$ supp class I & $1.47(0.18)$ & $2.87(0.09)$ \\
\hline$\Delta e x o R$ supp class II & $0.47(0.08)$ & $14.32(0.43)$ \\
\hline$\Delta$ exoRexoA supp & $2.25(0.30)$ & $0.52(0.12)$ \\
\hline
\end{tabular}

${ }^{\star} \mathrm{CV}$ absorbance of adherent biomass normalized to culture density.

$\dagger$ Anthrone absorbance normalized to culture density. 
Table 3. Disruption of exoR affects transcription of exoY and flgD genes

\begin{tabular}{|lrc|}
\hline Strain & \multicolumn{1}{c|}{ exo $_{\mathbf{N}^{*}}$} & \multicolumn{1}{c|}{ flgD $_{\mathbf{N}^{*}}$} \\
\hline C58 & $1.000 \pm 0.35$ & $1.000 \pm 0.40$ \\
C58 (pTWD100, $\mathrm{P}_{\text {lac }}::$ exoR) & $0.496 \pm 0.54$ & $1.225 \pm 0.27$ \\
exoR & $30.172 \pm 0.55$ & $0.024 \pm 0.26$ \\
AexoR (pTWD100, $\mathrm{P}_{\text {lac }}::$ exoR) & $1.071 \pm 0.23$ & $1.106 \pm 0.37$ \\
$\operatorname{chvG}$ & $0.417 \pm 0.12$ & $0.585 \pm 0.15$ \\
\hline
\end{tabular}

${ }^{\star}$ The range given for expression, relative to C58, encompasses one SD.

deficiency, indicating that succinoglycan is not required for biofilm formation (Table 2, Fig. 2g). The exoRexoA double mutant, despite its inability to overproduce succinoglycan, exhibited an adherence defect similar to that of the exoR single mutant (Fig. 2j). Although succinoglycan overproduction may contribute to the overall biofilm deficiency of the exoR mutant, it is clearly not the sole cause of the defect.

\section{Quantitative analysis of flow cell biofilms}

Biofilms of the exo mutant strains were grown in a oncethrough continuous-flow cell system and analysed using SDCM, and the COMSTAT analysis program (Heydorn et al., 2000). Microscopic examination of biofilm formation by $A$. tumefaciens C58 derivatives constitutively expressing GFP
$\left(\mathrm{pJZ383}, \mathrm{P}_{t a c}:: g f p\right)$ in flow cells was performed in real-time without disrupting the samples or ceasing flow. The autoCOMSTAT program provides quantitative, statistically robust analysis of biofilm structure via processing of image stacks from multiple fields of view within the flow cell. SDCM images show that the exoR mutant demonstrates a severe biofilm formation deficiency and few attached cells (Fig. 2e), in comparison with the wild-type C58 (Fig. 2b), while the exoA mutant was fully competent for biofilm formation (Fig. 2h). As with the static coverslip biofilms, the flow cell biofilms of the exoRexoA double mutant were similar to exoR mutant biofilms (Fig. $2 \mathrm{k}$ ). The biofilm deficiencies of both exoR and exoRexoA mutants were fully complemented by expression of plasmid-borne exoR (data not shown).

Quantitative COMSTAT analysis reveals statistically significant differences in such measurements as the amount of adherent biomass, average biofilm height and the maximum biofilm height for each strain (Table 4). These data support the qualitative assessment that the exoA mutant forms a wild-type biofilm, whereas the exoR and exoRexoA mutant strains are deficient in all measurements of biofilm formation that were quantified. These mutants occasionally bound as single cells or small cell clusters of five to six cells in height (Table 4), which rarely expanded further. Provision of plasmid-borne ExoR in either mutant background complemented the $\Delta$ exoR phenotype (Table 4). The overall flow cell phenotypes provide a more detailed view of attachment and biofilm structure
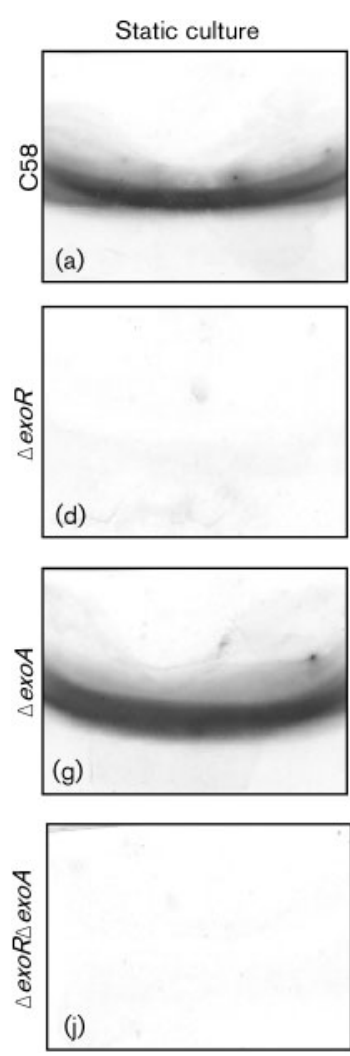
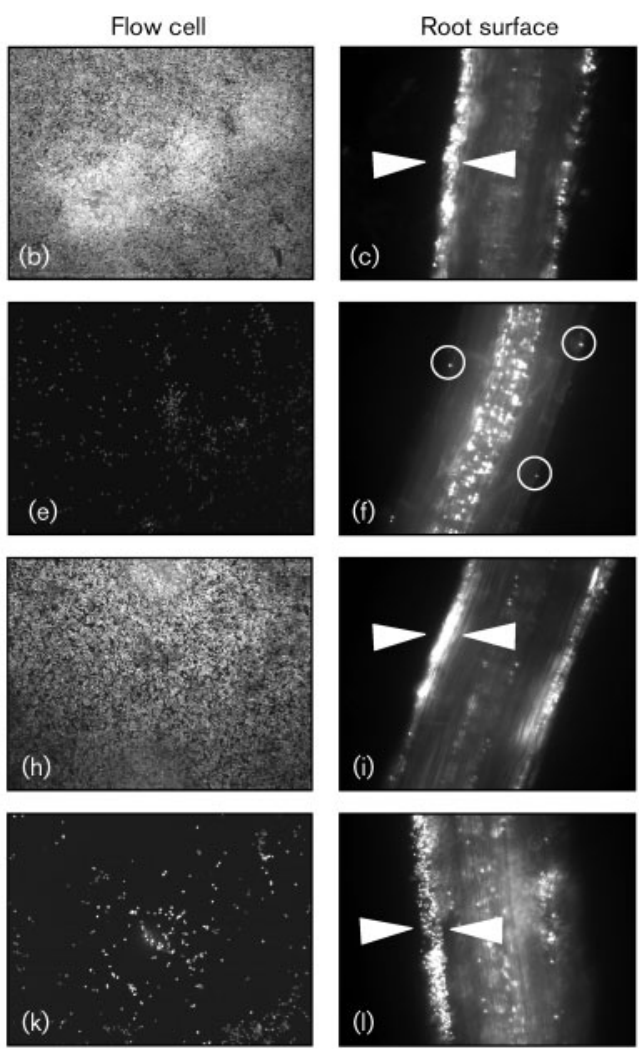

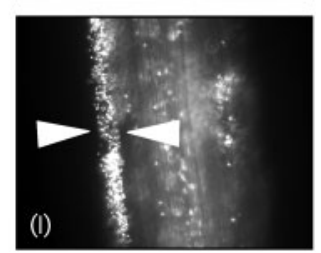

Fig. 2. Biofilm phenotypes of exoR and exoA mutants. Biofilms formed after $48 \mathrm{~h}$ static culture growth are shown in (a), (d), (g) and (j). (b), (e), (h) and (k) show biofilms grown under flow conditions for $72 \mathrm{~h}$. Surface attachment to cuttings of Arabidopsis thaliana roots are shown in (c), (f), (i) and (I). Individually bound cells are indicated by circles in ( $f$ ), while biofilms are indicated by arrowheads. Fluorescence of plant vasculature is due to autofluorescence, not microbial colonization of the vasculature. Wild-type cells ( $a, b$ and $c$ ) and $\Delta e x O A$ cells $(\mathrm{g}, \mathrm{h}$ and $\mathrm{i}$ ) are competent for biofilm formation under all conditions, while $\Delta \operatorname{exo} R$ ( $\mathrm{d}$, e and f) cultures do not form biofilms in any assay. $\triangle$ exoRexoA cells do not form biofilms on abiotic surfaces ( $j, k$ ), but are competent for plant tissue attachment (I). Confocal microscopy was performed using a Nikon TE-200 spinning disk confocal microscope at $\times 100$ magnification fitted with a Uniblitz shutter driver and an Orca ER camera (Hamamatsu) and Perkin-Elmer ULTRAVIEW software; epifluorescence microscopy was performed on a Nikon E800 at 60× magnification with METAMORPH software. 
Table 4. COMSTAT analysis of flow-cell biofilms over 4 days

Values are means $( \pm$ SEM) of 15 fields of view from three separate flow cells.

\begin{tabular}{|c|c|c|c|c|}
\hline Strain $^{*}$ & Time (h) & Biovolume/area $\dagger$ & Mean thickness $(\mu \mathrm{m})$ & Maximum thickness $(\mu \mathrm{m})$ \\
\hline \multirow[t]{2}{*}{ Wild-type } & 48 & $0.17(0.09)$ & $0.26(0.13)$ & $4.58(0.62)$ \\
\hline & 96 & $3.68(0.67)$ & $4.16(0.72)$ & $21.33(2.23)$ \\
\hline \multirow[t]{2}{*}{$\Delta e x o R$} & 48 & $0.00(0.00)$ & $0.00(0.00)$ & $3.00(0.22)$ \\
\hline & 96 & $0.06(0.01)$ & $0.01(0.00)$ & $6.33(0.42)$ \\
\hline \multirow[t]{2}{*}{$\triangle e x o A$} & 48 & $0.05(0.02)$ & $0.07(0.03)$ & $5.08(0.62)$ \\
\hline & 96 & $3.88(0.81)$ & $4.28(0.88)$ & $17.00(5.00)$ \\
\hline \multirow[t]{2}{*}{$\triangle \operatorname{exoRexoA}$} & 48 & $0.00(0.00)$ & $0.00(0.00)$ & $3.08(0.45)$ \\
\hline & 96 & $0.04(0.01)$ & $0.88(0.05)$ & $6.50(1.15)$ \\
\hline \multirow[t]{2}{*}{$\Delta e x o R, \mathrm{P}_{\text {lac }}::$ exoR } & 48 & $0.23(0.07)$ & $0.35(0.11)$ & $4.67(0.68)$ \\
\hline & 96 & $5.89(0.34)$ & $6.87(0.35)$ & $21.83(2.71)$ \\
\hline \multirow{2}{*}{$\Delta \operatorname{exoRexoA}, \mathrm{P}_{l a c}:: \operatorname{exoR}$} & 48 & $0.43(0.21)$ & $0.26(0.13)$ & $4.83(0.38)$ \\
\hline & 96 & $8.00(1.85)$ & $9.10(1.97)$ & $28.67(4.44)$ \\
\hline
\end{tabular}

${ }^{\star}$ All strains are derivatives of $A$. tumefaciens C58 carrying pJZ383 $\left(\mathrm{P}_{\text {tac }}:: g f p\right)$.

$\dagger \mu \mathrm{m}^{3}$ fluorescent material per $\mu \mathrm{m}^{2}$ surface attachment.

and are entirely consistent with the static culture phenotypes.

\section{ExoR is required for binding to plant root surfaces}

Plant-root-binding assays were performed to evaluate the influence of the exoR mutation and succinoglycan production on associations with plant tissues. Sterile segments of Arabidopsis thaliana roots were incubated with the A. tumefaciens derivatives harbouring the pJZ383 plasmid expressing GFP. Root segments were washed to remove loosely associated bacteria and examined under epifluorescence microscopy. Both C58 wild-type and exoA mutants were able to bind Arabidopsis thaliana root cuttings (Fig. 2c and i), but exoR mutants were dramatically deficient (Fig. 2f). Surprisingly, however, the exoRexoA double mutant bound efficiently to root tissue (Fig. 21). This finding illustrates that in contrast with results from abiotic surfaces, overproduction of succinoglycan is a dominant factor in the plant-binding deficiency of exoR mutants.

The exoR mutant's reduced propensity for root binding in suspension is not reflected in simple virulence assays in which A. tumefaciens is manually inoculated into plant wounds. When introduced onto sterile potato slices, the exoR mutant formed tumours indistinguishable in size and number from the wild-type (data not shown).

\section{ExoR can function independently of the ChvG- Chvl two component system}

The $S$. meliloti ExoR protein is secreted into the periplasm. Due to this unconventional location for a regulator, we began to consider possible intermediates through which $A$. tumefaciens ExoR might act. The first gene we targeted was $\operatorname{chvG}$, a two-component-type histidine kinase homologous to the S. meliloti gene exoS. In S. meliloti, ExoR is reported to function through interactions with ExoS and its cognate response regulator $\mathrm{ChvI}$, that together control production of multiple exopolysaccharides, including succinoglycan (Belanger et al., 2009). A point mutation that decreases ChvI activity (K214T) has been described that suppresses exoR mutant phenotypes in that system (Wells et al., 2007; Yao et al., 2004). Therefore, we created a $\operatorname{ch} v G$ mutation via Campbell integration in wild-type and the exoR, exo $A$ and exoRexoA mutant backgrounds to examine potential interactions in A. tumefaciens. All chvG mutants exhibited sensitivity to growth on LB medium, consistent with previous reports on A. tumefaciens (Charles \& Nester, 1993; Mantis \& Winans, 1993) and S. meliloti (Belanger et al., 2009). If exoR influences exopolysaccharide production exclusively through the ChvG-ChvI two component system, we would anticipate that a $\operatorname{chv} G$ null mutation would eliminate exopolysaccharide overproduction in an exoRchvG double mutant, consistent with observations in S. meliloti (Wells et al., 2007). In contrast with that hypothesis, the exoRchvG mutant retained elevated levels of exopolysaccharide production, although these were somewhat reduced relative to the exoR mutant (Table 2), indicating that ExoR can affect succinoglycan production independently of ChvG. In contrast with the hypermucoid exoR phenotype, we found that the $\operatorname{chv} G$ mutant was slightly reduced in exopolysaccharide production as measured by the anthrone assay. Furthermore, expression of the exoY gene is $60 \%$ reduced in the $c h v G$ mutant background, compared with a 30-fold derepression in the exoR background, indicating a modest role for ChvG in positively regulating exo gene expression (Table 3 ).

Additionally, we found that biofilm formation in the $\operatorname{ch} v G$ mutant not only occurs but is also, in fact, slightly increased compared with the wild-type (Table 2). The exoR mutation is also dominant over the $\operatorname{chv} G$ mutation in both 
the succinoglycan-positive and -negative backgrounds. This indicates that the exoR regulation of biofilm formation occurs independently of the $\operatorname{chv} G$ signal transduction pathway. Interestingly, the exoAchvG double mutant is somewhat reduced in biofilm formation, demonstrating that the modestly elevated $\operatorname{chv} G$ biofilm phenotype may be dependent on succinoglycan production. Thus, it appears that $\operatorname{chv} G$ has a mild negative influence on succinoglycan production - the mutant yields a slight increase in biofilm formation - but that this phenotype is independent of regulation by ExoR.

\section{ExoR mutants exhibit reduced motility}

Examination of exoR mutants revealed that they were severely deficient in swimming motility, forming a small ring relative to the wild-type after 4-7 days incubation on swim agar ( $0.25 \%$ agar) (Fig. $3 \mathrm{a}$ and b). The exoA mutant was proficient for swimming motility with no visible mucoidy (Fig. 3c). Close examination of the exoR mutant swim plate revealed an accumulation of mucoid material on the surface at the site of inoculation, and it was plausible that this mucoid material inhibited motility. The exoRexoA double mutant, however, exhibited similarly attenuated motility, indicating that the motility deficiency of the exoR mutant is independent of succinoglycan overproduction (Fig. 3d). The exoR and exoRexoA mutant motility phenotypes were fully complemented by introduction of the $\mathrm{P}_{\text {lac }}$ : : exoR plasmid (Fig. 3e and f). The swim plate phenotypes were consistent with microscopic examination of cells that were stained to visualize flagella, in which exoR and exoRexoA mutant cultures had predominantly aflagellate cells, while the exoA mutant was flagellated equivalent to wild-type (data not shown). These findings suggest that ExoR independently regulates both motility and succinoglycan production.

The absence of flagella suggested a defect in synthesis or assembly of flagellin. Flagellin levels were examined by immunoblotting using cross-reactive antibody raised against Caulobacter crescentus flagella. While the wild-type strain produced easily observable levels of flagellin, the protein was undetectable in the $\triangle$ exoR mutant, similar to aflagellate $\triangle$ flgE (flagellar hook) and $\triangle$ flaABC (flagellin) mutants (Fig. 3j).

qPCR was used to examine expression levels of $f l g D$, the scaffold for flagellar hook assembly. Consistent with swim plate phenotypes and flagellin levels, $f l g D$ expression is nearly abolished in the exoR mutant relative to the wildtype. The plasmid-borne ExoR restores wild-type expression levels of $f \lg D$. A modest decrease in $f l g D$ expression is observed in a chvG mutant as well (Table 3).

Although the exoR and exoRexoA deletion mutants were clearly reduced in flagellation, a small number of flagella were always observed, consistent with the reduced but significant swim rings observed on motility agar. The swim plate environment appears to efficiently select for sup-
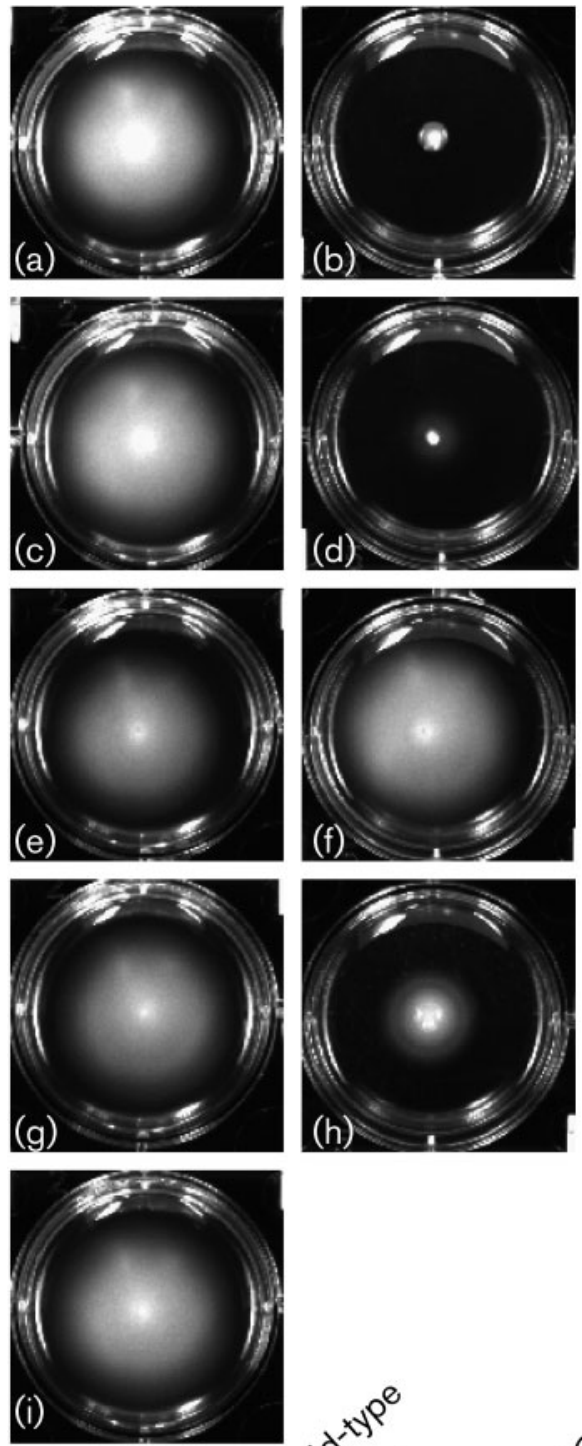

(j)

$37830 \mathrm{Da}$ Flagellin

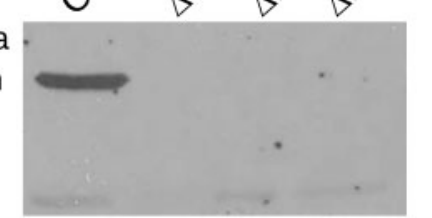

Fig. 3. ExoR null mutants are reduced in swimming motility and production of flagellin. Wild-type cells (a) are fully motile in $0.25 \%$ swim agar, while $\Delta$ exoR mutants are non-motile (b). Succinoglycan- $\triangle$ exo $A$ mutants have wild-type motility (c), but $\triangle$ exoRexoA mutants are deficient in motility (d), demonstrating that succinoglycan is not required for motility and that ExoR acts independently to regulate succinoglycan production and motility. Both the $\Delta e x o R(\mathrm{e})$ and $\Delta e x o R e x o A(\mathrm{f})$ non-motile phenotypes are complemented by addition of $\mathrm{P}_{\text {lac }}:$ : exoR. Class I suppressors of $\triangle$ exoR show wild-type motility (g), while class II suppressors show a partial restoration of motility (h). Suppressors of $\triangle$ exoRexoA are fully motile (i). Western blotting shows a reduction of flagellin in $\Delta$ exoR mutants compared with wild-type C58 (j). 
pressor mutants. In each repetition of the swim assay, suppressors of the motility-deficient phenotype of the exoR and exoRexoA strains were observed at the edges of the swim ring. The suppressors of the exoR mutation fell into two classes: class I suppressors form dry, non-mucoid colonies, are apparently wild-type for motility and exopolysaccharide production, and form wild-type biofilms in the static culture coverslip assay (Fig. 3g, Table 2). Class II suppressors form mucoid colonies and accordingly produce exopolysaccharide at levels as high as exoR mutants. Class II mutants exhibit motility levels intermediate between the non-motile exoR mutants and the wild-type cells, but are still unable to form biofilms on coverslips (Fig. $3 \mathrm{~h}$, Table 2). All observed suppressors of the exoRexoA mutant exhibited dry colony morphologies, wild-type motility, and improved biofilm formation (Fig. $3 i$, Table 2). Introduction of a plasmid-borne copy of exoR into the non-mucoid, motile and biofilm-proficient class I mutants had no significant effect on these phenotypes (data not shown). In contrast, introduction of the exoR plasmid into the class II suppressors (mucoid, partially motile and biofilm-deficient) complemented all of these phenotypes to wild-type levels. These observations are consistent with the class I suppressors already functioning as nearly wild-type, and therefore not being affected by the presence of exoR. The class II suppressor mutations partially correct the motility deficiency, and thus provision of exoR restores full motility as well as correcting the remaining mutant phenotypes.

Together, these data suggest that we have identified suppressor mutations at two discrete points in the ExoRdependent regulatory pathway. A previously reported suppressor of the $S$. meliloti exoR mutant mapped to $\operatorname{chvI}$ (Wells et al., 2007). As such, we conducted sequence analysis of the chvGI region, including its upstream sequences, in the original $A$. tumefaciens exoR mutant and its suppressors. This analysis revealed all of these sequences to be identical to the corresponding sequences in wild-type C58. Thus, the isolated suppressor mutations must have occurred outside the chvGI region, and ChvG and ChvI are not required for ExoR regulation of these functions.

\section{DISCUSSION}

A. tumefaciens is a widely prevalent soil microbe, but is best known as a plant pathogen. Surface attachment is a required early step in the initiation of the infection process, and likewise is required for biofilm formation. In this study, we performed a screen for biofilm-deficient mutants of A. tumefaciens C58 and isolated two independent mutations in the exoR gene. ExoR is a regulator known to be required for nodulation through control of succinoglycan in the nitrogen-fixing alfalfa symbiont $S$. meliloti (Doherty et al., 1988; Reed et al., 1991). We found that A. tumefaciens C58 exoR null mutants are deficient in surface attachment, and therefore do not form biofilms on either plant tissues or abiotic surfaces. ExoR is a negative regulator of succinoglycan synthesis, and an exoR null mutant overproduces this exopolysaccharide. Succinoglycan overproduction requires the exo genes, and the expression of at least one of these genes is significantly derepressed in the exoR mutant. The attachment deficiency on abiotic model surfaces is not simply due to succinoglycan overproduction, but apparently involves other functions influenced by ExoR. At least one other function clearly under ExoR regulatory control is motility; exoR mutants exhibit reduced motility that corresponds to a loss of flagellation, and markedly reduced flagellar gene expression.

The motility deficiency of the exoR mutant is unlikely to be entirely responsible for its biofilm deficiency. In a separate study, we investigated the role of motility during $A$. tumefaciens biofilm formation (Merritt et al., 2007). Although markedly reduced for biofilm formation in non-flowing systems, an aflagellate A. tumefaciens $f l g E$ mutant is highly competent for biofilm formation in flow cells. To exclude the possibility of a synthetic effect between motility and succinoglycan production on biofilm formation, we examined static culture biofilms of a flgE exoA double mutant. The double mutant exhibited the nonmotile phenotype of the flgE parent strain, the succinoglycan production deficiency of the exoA parent strain, and was identical in biofilm formation to the flgE parent strain (data not shown). In contrast with the non-motile flgE mutant, the exoR mutant shows similarly compromised attachment under both non-flowing and flowing conditions. Also, although the exoRexoA mutant is reduced in swimming motility and abiotic surface attachment, it does form biofilms on plant tissue, indicating that its motility deficiency is not the underlying cause of its biofilm phenotype.

ExoR is broadly conserved across the alphaproteobacteria, including the plant symbionts Rhizobium etli, Rhizobium leguminosarum and Mesorhizobium loti, in animal pathogens such as Brucella suis and Brucella abortus, and even in free-living bacteria such as Rhodopseudomonas palustris. The amino acid sequence is highly conserved, with 40 invariant residues spread throughout the length of the sequence (Supplementary Fig. S1). In all homologues, a conserved secretion signal motif is located at the $\mathrm{N}$ terminus of the translation product. Tetratricopeptide repeat domains, and the closely related SEL-1 domains that extend through the ExoR sequence are implicated in protein-protein interactions (Mittl \& Schneider-Brachert, 2007). This suggests that ExoR exerts its regulatory effects via interaction with other regulatory or signal transduction system(s). The sequence similarities imply an analogous biochemical function(s) for ExoR in each microbial system.

ExoR has been extensively investigated in S. meliloti, where it was originally identified as a repressor of succinoglycan production (Reed et al., 1991). Our results reveal that ExoR is also a negative regulator of succinoglycan production via 
exo gene repression in A. tumefaciens. In both bacteria, mutation of exoR causes hypermucoidy and an increase in exo gene expression (Reed et al., 1991; Chen et al., 2008) (Table 3). Additionally, ExoR appears to be required for flagellar motility in both organisms (Yao et al., 2004). However, other phenotypes resulting from disruption of exoR in the two bacteria are strikingly different. Succinoglycan is required for $S$. meliloti to form functional symbiotic relationships with host legumes (Leigh et al., 1987), and the exoR mutant is also symbiotically deficient. In contrast, succinoglycan is dispensable for crown gall disease in A. tumefaciens (Cangelosi et al., 1987) and we find that exoR mutants form tumours efficiently upon manual inoculation of model plants such as potato. Wells et al. (2007) reported that an exoR:: Tn5 mutation, which results in high-level succinoglycan synthesis, increases $S$. meliloti biofilm formation on abiotic surfaces. Disruption of succinoglycan synthesis (exoY:: Tn5) correspondingly decreased biofilm formation in a similar assay (Fujishige et al., 2006). Our isolation of two separate A. tumefaciens exoR transposon mutants based on their inability to form biofilms, which were found to overproduce succinoglycan, directly contrasts with these phenotypes. Consistent with this, an A. tumefaciens mutant unable to synthesize succinoglycan (exoA disruption) is unaffected with respect to biofilm formation. However, a more recent report for $S$. meliloti is in agreement with our findings, demonstrating that succinoglycan-deficient mutants form normal biofilms, but that exopolysccharide II (galactoglucan) is required (Rinaudi \& Gonzalez, 2009). Another difference is the observation that the $S$. meliloti exoR:: Tn 5 mutant is auxotrophic, while the A. tumefaciens exoR mutant strains are uniformly prototrophic.

S. meliloti ExoR associates with and inhibits the ExoS sensor kinase, and through it, the response regulator ChvI (Chen et al., 2008; Wells et al., 2007). ExoS and ChvI control exopolysaccharide production and apparently inhibit motility in S. meliloti (Belanger et al., 2009; Wells et al., 2007). We examined an A. tumefaciens $\operatorname{chv} G$ mutant and observed decreased expression of exoY and a slight decrease in exopolysaccharide production, indicating that ChvG exerts a mild positive regulation of exopolysaccharide levels. A $\operatorname{chv} G$ mutation also slightly increases $A$. tumefaciens biofilm formation. We have observed that a $\operatorname{ch} v G$ mutation modestly decreases flagellar gene expression (i.e., the $f \lg D$ transcript), although this does not affect motility (data not shown).

S. meliloti exoR mutants are reported to be non-motile and to exhibit an elevated biofilm phenotype. Mutations that decrease ChvI activity can suppress the motility defect of an exoR mutant and restore wild-type biofilm levels (Wells et al., 2007). In contrast, in A. tumefaciens, ExoR regulation of biofilm formation, motility and exopolysaccharide production appears not to require the ChvG (ExoS)/ChvI twocomponent system. Loss of a functional ChvG/I does not restore wild-type biofilm, or exopolysaccharide phenotypes to an exoR mutant. In further contrast with $S$. meliloti reports, mutation of ChvG/I in an A. tumefaciens exoR mutant does not restore motility (data not shown). Therefore, we conclude that A. tumefaciens ExoR must be able to function through a signalling pathway distinct from ChvG/I.

One way to define such a pathway is by isolating mutants in which exoR mutant phenotypes are suppressed. The two classes of exoR mutant suppressors we have isolated thus far indicate a branched regulatory pathway. Class I suppressors cause reversion to wild-type levels of both motility and exopolysaccharide production, and these suppressors regain competence for biofilm formation. Class II suppressors still overproduce exopolysaccharide and remain mucoid, but have regained intermediate motility compared to the wild-type. The simplest explanation is that the intermediate motility of the class II suppressors is due to excess exopolysaccharide, blocking motility by increasing local viscosity. Class II suppressors remain incompetent for biofilm formation, another indication that the biofilm deficiency of the exoR mutant is not solely due to the loss of motility. The single class of suppressors (Class I-like) isolated for the exoRexoA mutant have regained wild-type levels of motility and biofilm formation, and remain succinoglycan-deficient (due to the exoA mutation). Provision of wild-type exoR in both class I and class II suppressor mutants leads to phenotypes consistent with the current model. Similar suppressors of exoR mutations in $S$. meliloti consistently map to the exoS and chvI genes (Wells et al., 2007), reflecting the ExoRExoS interaction. In the A. tumefaciens suppressors described here, the $c h v G$ and $c h v I$ genes are not mutated.

A mechanism by which ExoR functions via its inhibition of ExoS/ChvI, while strongly supported in S. meliloti, does not seem to apply to the phenotypes we have examined in $A$. tumefaciens. It is possible that ExoR influences a single regulatory pathway to exert the wide variety of effects we observe. A cytoplasmic regulatory circuit implicated in control of exopolysaccharide production and motility in $S$. meliloti includes the zinc finger protein MucR, the LuxRtype regulators VisN/R and their interacting partner Rem (Bahlawane et al., 2008). Homologues of each of these regulators are present in A. tumefaciens $\mathrm{C} 58$, and these may be indirect targets for ExoR regulation. Indeed, expression of the MucR homologue Ros is elevated in the $\Delta$ exoR mutant, which may account for the hypermucoidy of this mutant (A. D. Tomlinson and C. Fuqua, unpublished data). Alternatively, ExoR may be influencing multiple, discrete pathways, including ChvG/I, some of which impinge upon the process of surface attachment. Finally, a more indirect role is possible. For example, given its location in cells, ExoR may serve to sense the state of the periplasm and thereby influence multiple aspects of cellular physiology. Future experiments will discriminate between these models by identifying the partners with which ExoR interacts, as well as the downstream targets of ExoR regulation. These studies will also elucidate whether ExoR functions in response to specific environmental cues or is a 
required but non-modulated component of these regulatory pathways.

\section{ACKNOWLEDGEMENTS}

We wish to acknowledge José Rodriguez, Rania Rizk and Melanie Lawler for valuable input on this project. Yves Brun generously provided the anti-flagellin antibody. Thanks also to Juan González for the anthrone assay, and both Juan González and Lois Banta for helpful discussions. The Indiana University Light Microscopy Imaging Center provided valuable assistance and microscopy for this study. Daniel Kearns provided useful suggestions for the manuscript. A.D.T. and B.R.-H. were appointees on the Indiana University Genetics, Molecular and Cellular Sciences Training Grant T32GM007757. This project was supported by National Institutes of Health grant RO1-GM080546 (C.F.) and through a grant from the Indiana University META-Cyt program funded in part by a major endowment from the Lilly Foundation (C.F.).

\section{REFERENCES}

Ausmees, N., Jacobsson, K. \& Lindberg, M. (2001). A unipolarly located, cell-surface-associated agglutinin, RapA, belongs to a family of Rhizobium-adhering proteins (Rap) in Rhizobium leguminosarum bv. trifolii. Microbiology 147, 549-559.

Bahlawane, C., Mclntosh, M., Krol, E. \& Becker, A. (2008). Sinorhizobium meliloti regulator MucR couples exopolysaccharide synthesis and motility. Mol Plant Microbe Interact 21, 1498-1509.

Barbosa, R. L. \& Benedetti, C. E. (2007). BigR, a transcriptional repressor from plant-associated bacteria, regulates an operon implicated in biofilm growth. J Bacteriol 189, 6185-6194.

Belanger, L., Dimmick, K. A., Fleming, J. S. \& Charles, T. C. (2009). Null mutations in Sinorhizobium meliloti exoS and chvI demonstrate the importance of this two-component regulatory system for symbiosis. Mol Microbiol 74, 1223-1237.

Blatch, G. L. \& Lassle, M. (1999). The tetratricopeptide repeat: a structural motif mediating protein-protein interactions. Bioessays 21, 932-939.

Branda, S. S., Vik, S., Friedman, L. \& Kolter, R. (2005). Biofilms: the matrix revisited. Trends Microbiol 13, 20-26.

Cangelosi, G. A., Hung, L., Puvanesarajah, V., Stacey, G., Ozga, D. A., Leigh, J. A. \& Nester, E. W. (1987). Common loci for Agrobacterium tumefaciens and Rhizobium meliloti exopolysaccharide synthesis and their roles in plant interactions. J Bacteriol 169, 2086-2091.

Charles, T. C. \& Nester, E. W. (1993). A chromosomally encoded twocomponent sensory transduction system is required for virulence of Agrobacterium tumefaciens. J Bacteriol 175, 6614-6625.

Chen, E. J., Sabio, E. A. \& Long, S. R. (2008). The periplasmic regulator ExoR inhibits ExoS/ChvI two-component signalling in Sinorhizobium meliloti. Mol Microbiol 69, 1290-1303.

Chesnokova, O., Coutinho, J. B., Khan, I. H., Mikhail, M. S. \& Kado, C. I. (1997). Characterization of flagella genes of Agrobacterium tumefaciens, and the effect of a bald strain on virulence. Mol Microbiol 23, 579-590.

Christie, P. J., Atmakuri, K., Krishnamoorthy, V., Jakubowski, S. \& Cascales, E. (2005). Biogenesis, architecture, and function of bacterial type IV secretion systems. Annu Rev Microbiol 59, 451485.

Cormack, B. P., Valdivia, R. H. \& Falkow, S. (1996). FACS-optimized mutants of the green fluorescent protein (GFP). Gene 173, 33-38.
D’Andrea, L. D. \& Regan, L. (2003). TPR proteins: the versatile helix. Trends Biochem Sci 28, 655-662.

Danhorn, T., Hentzer, M., Givskov, M., Parsek, M. \& Fuqua, C. (2004). Phosphorous limitation enhances biofilm formation of the plant pathogen Agrobacterium tumefaciens through the PhoR-PhoB regulatory system. J Bacteriol 186, 4492-4501.

Dardanelli, M., Angelini, J. \& Fabra, A. (2003). A calcium-dependent bacterial surface protein is involved in the attachment of rhizobia to peanut roots. Can J Microbiol 49, 399-405.

Dennis, J. J. \& Zylstra, G. J. (1998). Plasposons: modular self-cloning minitransposon derivatives for rapid genetic analysis of Gramnegative bacterial genomes. Appl Environ Microbiol 64, 2710-2715.

Doherty, D., Leigh, J. A., Glazebrook, J. \& Walker, G. C. (1988). Rhizobium meliloti mutants that overproduce the $R$. meliloti acidic Calcofluor-binding exopolysaccharide. J Bacteriol 170, 4249-4256.

Escobar, M. A. \& Dandekar, A. M. (2003). Agrobacterium tumefaciens as an agent of disease. Trends Plant Sci 8, 380-386.

Fujishige, N. A., Kapadia, N. N., De Hoff, P. L. \& Hirsch, A. M. (2006). Investigations of Rhizobium biofilm formation. FEMS Microbiol Ecol 56, 195-206.

Fuqua, C. (2008). Agrobacterium-host attachment and biofilm formation. In Agrobacterium: from Biology to Biotechnology, pp. 243-277. Edited by T. Tzfira \& V. Citovsky. New York, NY: Springer.

Gelvin, S. B. (2003). Agrobacterium-mediated plant transformation: the biology behind the "gene-jockeying" tool. Microbiol Mol Biol Rev 67, 16-37.

Goodner, B., Hinkle, G., Gattung, S., Miller, N., Blanchard, M., Qurollo, B., Goldman, B. S., Cao, Y., Askenazi, M. \& other authors (2001). Genome sequence of the plant pathogen and biotechnology agent Agrobacterium tumefaciens C58. Science 294, 2323-2328.

Hall-Stoodley, L., Costerton, J. W. \& Stoodley, P. (2004). Bacterial biofilms: from the natural environment to infectious diseases. Nat Rev Microbiol 2, 95-108.

Heydorn, A., Toftgaard Nielsen, A., Hentzer, M., Sternberg, C., Givskov, M., Kjaer Ersboll, B. \& Molin, S. (2000). Quantification of biofilm structures by the novel computer program COMSTAT. Microbiology 146, 2395-2407.

Kalogeraki, V. S. \& Winans, S. C. (1997). Suicide plasmids containing promoterless reporter genes can simultaneously disrupt and create fusions to target genes of diverse bacteria. Gene 188, 69-75.

Kaniga, K., Delor, I. \& Cornelis, G. R. (1991). A wide-host-range suicide vector for improving reverse genetics in Gram-negative bacteria: inactivation of the blaA gene of Yersinia enterocolitica. Gene 109, 137-141.

Koutsoudis, M. D., Tsaltas, D., Minogue, T. D. \& von Bodman, S. B. (2006). Quorum-sensing regulation governs bacterial adhesion, biofilm development, and host colonization in Pantoea stewartii subspecies stewartii. Proc Natl Acad Sci U S A 103, 5983-5988.

Laus, M. C., Logman, T. J., Lamers, G. E., Van Brussel, A. A., Carlson, R. W. \& Kijne, J. W. (2006). A novel polar surface polysaccharide from Rhizobium leguminosarum binds host plant lectin. Mol Microbiol 59, 1704-1713.

Leigh, J. A., Reed, J. W., Hanks, J. F., Hirsch, A. M. \& Walker, G. C. (1987). Rhizobium meliloti mutants that fail to succinylate their calcofluor-binding exopolysaccharide are defective in nodule invasion. Cell 51, 579-587.

Lim, B., Beyhan, S. \& Yildiz, F. H. (2007). Regulation of Vibrio polysaccharide synthesis and virulence factor production by CdgC, a GGDEF-EAL domain protein, in Vibrio cholerae. J Bacteriol 189, 717729.

Mantis, N. J. \& Winans, S. C. (1993). The chromosomal response regulatory gene chvI of Agrobacterium tumefaciens complements an 
Escherichia coli phoB mutation and is required for virulence. J Bacteriol 175, 6626-6636.

Matthysse, A. G., Yarnall, H., Boles, S. B. \& McMahan, S. (2000). A region of the Agrobacterium tumefaciens chromosome containing genes required for virulence and attachment to host cells. Biochim Biophys Acta 1490, 208-212.

Merritt, P. M., Danhorn, T. \& Fuqua, C. (2007). Motility and chemotaxis in Agrobacterium tumefaciens surface attachment and biofilm formation. J Bacteriol 189, 8005-8014.

Mersereau, M., Pazour, G. J. \& Das, A. (1990). Efficient transformation of Agrobacterium tumefaciens by electroporation. Gene 90, 149-151.

Miller, J. F., Mekalanos, J. J. \& Falkow, S. (1989). Coordinate regulation and sensory transduction in the control of bacterial virulence. Science 243, 916-922.

Mittl, P. R. \& Schneider-Brachert, W. (2007). Sel1-like repeat proteins in signal transduction. Cell Signal 19, 20-31.

Morris, D. L. (1948). Quantitative determination of carbohydrates with Dreywood's anthrone reagent. Science 107, 254-255.

Nair, G. R., Liu, Z. \& Binns, A. N. (2003). Reexamining the role of the accessory plasmid pAtC58 in the virulence of Agrobacterium tumefaciens strain C58. Plant Physiol 133, 989-999.

O’Toole, G. A., Pratt, L. A., Watnick, P. I., Newman, D. K., Weaver, V. B. \& Kolter, R. (1999). Genetic approaches to study of biofilms. Methods Enzymol 310, 91-109.

Ramey, B. E., Matthysse, A. G. \& Fuqua, C. (2004). The FNR-type transcriptional regulator SinR controls maturation of Agrobacterium tumefaciens biofilms. Mol Microbiol 52, 1495-1511.

Reed, J. W., Glazebrook, J. \& Walker, G. C. (1991). The exoR gene of Rhizobium meliloti affects RNA levels of other exo genes but lacks homology to known transcriptional regulators. J Bacteriol 173, 37893794.

Reuber, T. L. \& Walker, G. C. (1993). Biosynthesis of succinoglycan, a symbiotically important exopolysaccharide of Rhizobium meliloti. Cell 74, 269-280.

Rinaudi, L. V. \& Gonzalez, J. E. (2009). The low-molecular-weight fraction of exopolysaccharide II from Sinorhizobium emliloti is a crucial determinant of biofilm formation. J Bacteriol 191, 7216-7224.
Russo, D. M., Williams, A., Edwards, A., Posadas, D. M., Finnie, C., Dankert, M., Downie, J. A. \& Zorreguieta, A. (2006). Proteins exported via the PrsD-PrsE type I secretion system and the acidic exopolysaccharide are involved in biofilm formation by Rhizobium leguminosarum. J Bacteriol 188, 4474-4486.

Sambrook, J., Fritsch, E. \& Maniatis, T. (1989). Molecular Cloning: a Laboratory Manual, 2nd edn. Cold Spring Harbor, NY: Cold Spring Harbor Laboratory.

Sciaky, D., Montoya, A. L. \& Chilton, M.-D. (1978). Fingerprints of Agrobacterium Ti plasmids. Plasmid 1, 238-253.

Smit, G., Logman, T. J. J., Boerrigter, M. E. T. I., Kijne, J. W. \& Lugtenberg, B. J. J. (1989). Purification and partial characterization of the $\mathrm{Ca}^{2+}$ dependent adhesin from Rhizobium leguminosarum biovar viciae, which mediates the first step in attachment of Rhizobiaceae cells to plant root hair tips. J Bacteriol 171, 4054-4062.

Tempé, J., Petit, A., Holsters, M., Van Montagu, M. \& Schell, J. (1977). Thermosensitive step associated with transfer of the Ti plasmid during conjugation: possible relation to transformation in crown gall. Proc Natl Acad Sci U S A 74, 2848-2849.

Wells, D. H., Chen, E. J., Fisher, R. F. \& Long, S. R. (2007). ExoR is genetically coupled to the ExoS-ChvI two-component system and located in the periplasm of Sinorhizobium meliloti. Mol Microbiol 64, 647-664.

Wood, D. W., Setulab, J. C., Kaul, R., Monks, D. E., Kitajima, J. P., Okura, V. K., Zhou, Y., Chen, L., Wood, G. E. \& other authors (2001). The genome of the natural genetic engineer Agrobacterium tumefaciens C58. Science 294, 2317-2323.

Woodcock, D. M., Crowther, P. J., Doherty, J., Jefferson, S., DeCruz, E., Noyer-Weidner, M., Smith, S. S., Michael, M. Z. \& Graham, M. W. (1989). Quantitative evaluation of Escherichia coli host strains for tolerance to cytosine methylation in plasmid and phage recombinants. Nucleic Acids Res 17, 3469-3478.

Yao, S. Y., Luo, L., Har, K. J., Becker, A., Ruberg, S., Yu, G. O., Zhu, J. B. \& Cheng, H. P. (2004). Sinorhizobium meliloti ExoR and ExoS proteins regulate both succinoglycan and flagellum production. J Bacteriol 186, 6042-6049.

Edited by: M. F. Hynes 